\title{
Use of augmented reality in the usability evaluation of products
}

\author{
Fernanda Faust ${ }^{\mathrm{a}, 1}$, Giorgia Roepke ${ }^{\mathrm{a}}$, Tiago Catecati ${ }^{\mathrm{a}}$, Fernanda Araujo ${ }^{\mathrm{b}}$, Marcelo Gitirana Gomes \\ Ferreira $^{\mathrm{a}}$ and Deise Albertazzi ${ }^{\mathrm{c}}$ \\ ${ }^{a}$ Departamento de Design, Universidade do Estado de Santa Catarina, Campus Itacorubi, Santa Catarina, Brasil. \\ bepartamento de Engenharia de Produção e Sistemas, Universidade Federal de Santa Catarina, Campus \\ Trindade, Florianópolis, Santa Catarina, Brasil \\ ${ }^{c}$ Departamento de Design, Universidade Federal do Paraná, Rua General Carneiro, 460, $8^{\circ}$ andar, Curitiba, \\ Paraná, Brasil.
}

\begin{abstract}
Many characteristics of a product are evaluated during the development phase, such as function, aesthetics and manufacturing. The conception phase is supported by drawings and computer graphics, followed by physical prototypes that help evaluating the product features. The augmented reality technology has a great potential to assist the designers in this phase, reducing the development process time and costs and improving the quality of the evaluation. This paper presents a study that intends to investigate the suitability of using augmented reality to evaluate the usability of electronic products.
\end{abstract}

Keywords: Augmented reality, product development, evaluation.

1.

\section{Introduction}

During the various phases of the product design several characteristics are evaluated, such as function, use, aesthetics, manufacturability, maintainability, and so on. These evaluations are important since they subsidize and impact the decisions taken by the project team. It is essential to have methods and tools to ensure a good evaluation during the conception phase of the design process. The early phases of the development process are those that most affect the quality and the cost of the product as well as their time to market - key factors for the success of the product in the market $[3,7,9]$.

In the industrial design process, the product is designed with the support of manual sketches and computer graphics (two or three-dimensional models). Later, physical prototypes (functional or non functional mock-ups) are built in order to evaluate some of the important aspects of the product. These physical prototypes, due to their high costs and time to build, increase the costs and the lead-time of the project. This usually has a negative repercussion on the competitiveness of the product when launched in the market.

Today, virtual prototypes are already used to simulate and evaluate some technical aspects of the products. Among many examples that could be presented, the aerodynamic performance of vehicles, aircraft and even buildings can be evaluated by using computer simulation, replacing the costly wind tunnels, which requires a physical prototype of the product.

In this context - the replacement or supplementation of physical simulations and assessments by the virtual based technologies - the augmented reality (AR) arises with the potential to help designers to evaluate several aspects of the product. Augmented reality can be defined as the superposition of virtual objects (computer generated images, texts, sounds etc.) on the real environment of the user. This technology can reduce substantially the costs and the lead-time, while maintaining or even enhancing the quality of the evaluations [6]. A great

\footnotetext{
${ }^{1}$ fernandagfaust@gmail.com
} 
advantage the AR has against the $\mathrm{VR}$ is the possibility to touch a physical object.

This paper presents a research that intends to investigate the suitability of using the augmented reality to assist the design team to evaluate some ergonomic aspects of electronic products. Virtual prototypes of the product will be analyzed in comparison with traditional physical prototype in the evaluation of the usability of consumer electronics.

\section{Usability}

According to the ISO 9241-11, the usability can be defined as the "extent to which a product can be used by specified users to achieve specified goals with effectiveness, efficiency and satisfaction in a specified context of use" [4]. To better understand this definition, it is worth clarifying the meaning of the terms effectiveness, efficiency and satisfaction. According to [11]:

- Effectiveness: refers to the extent to which a goal is achieved or a task is performed.

- Efficiency: refers to the amount of effort required to achieve a goal. The less effort, greater efficiency.

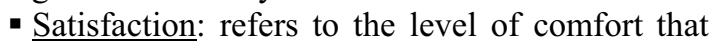
users feel when using a product and also the level of product acceptance by users to achieve their goals.

The definition makes it clear that usability is not an intrinsic property of the product itself. It depends on the specific context of use: who is using the product, for what purpose and where. For workrelated products, such as in a lathe, the effectiveness and efficiency tend to be the more important than the satisfaction. The opposite situation occurs with products related to entertainment such as games and cell phones.

A model for the usability of products and systems is presented by [11] and includes five components:

- Guessability: effectiveness, efficiency and satisfaction with which specific users can perform certain tasks with a particular product when they use it at the first time.

- Learnability: effectiveness, efficiency and satisfaction with which specific users can achieve specific levels of performance in certain tasks with a product, having already performed these tasks before.

- Experienced user performance: effectiveness, efficiency and satisfaction with which expert users can perform certain tasks with a particular product.

- System potential: effectiveness, efficiency and satisfaction with which the user could accomplish certain tasks with a product.

- Re-usability: effectiveness, efficiency and satisfaction with which specified users can perform certain tasks after having them done after a long period of time.

There are many methods that can be used to evaluate the usability of products (software and systems). [5] groups these methods into three broad categories: testing, inspections, and inquiries. In the testing, representative users perform typical tasks using the product or system (or its prototype) and the evaluators use the results to analyze how the product or system interface supports users to realize the tasks. During the inspections, some usability experts analyze the usability aspects of the product interface with the user. With observations and questionnaires, the evaluators obtain information about the positive and negative features of the product and information about the user needs by talking and observing them in their routine or asking them to answer orally or written questions.

The Think Aloud Protocol stands out among the user-based methods. From the Think Aloud Protocol derives many other methods that result in qualitative data. For gathering quantitative data, it is usually used performance testing. Among the expert-based evaluations, we highlight the Heuristic Evaluation and Cognitive Walkthrough methods.

The selection of a particular method for a usability evaluation is guided by several factors. First, we must determine the objectives of the evaluation, so we can identify all possible methods that can provide the data we need. An important factor in selecting a method is the number of participants, and usability experts, that will be needed. The required metrics are defined according to the goals of the evaluation. According to [2], time, costs and confidence of the results are among the factors that influence the process of choosing a usability method. According to [8], the desired output guides the choice of method because it directs the selection according to the time available for application and analysis.

\section{Augmented reality}

According to [1], even though the augmented reality (AR) is not a new technology, two major 
technological advances made possible its application in both sophisticated and popular platforms. First, the higher processing speed of modern personal computers, which allowed the integration, in realtime, of video and interactive virtual environments. The second advance is the increased network bandwidth that allows computers to transfer images and other data with greater efficiently.

The AR uses computational techniques to generate, position and show virtual objects integrated into realworld scenario, while the augmented virtuality uses computational techniques to capture real elements and rebuild them as realistic virtual objects, placing them in virtual worlds and allowing its interaction with the environment [1].

There are several ways in which the augmented reality is being used at research laboratories, industries, and also in the market, in the reach of consumers. The following are the most widespread forms of AR and also those that presents a greater potential for future use. Other less common and widespread technology for the generation of AR some of which are still under development - may be found in authors such as [10] or [12].

Desktop AR - or screen-based video see-through displays AR [10] - is one of the most popular and inexpensive way to implement $\mathrm{AR}$. It only requires a webcam, a personal computer and software to generate the augmented reality - some open source AR program are available to download in the Internet.

The AR with Head Mounted Displays (HMD) are divided into two types, depending on how the virtual images are added to the real world scene. The video see-through type works on a similar way as a computer display, however it covers much of the users visual field and promotes a vision alignment with the displays. The see-through optical has lenses partially transmissible, so that the user can look through them and see the real world. These lenses are also partially reflective, being possible to see the virtual images reflected on them [10].

The AR with Head Up Display (HUD), was initially developed for use in fighter aircraft, providing information for the pilot without it having to look away from the target in front of the aircraft. Today it is being used also in cars, providing information from the instrument panels. The HUDs used in aircraft and automobiles work on a similar way to the optical see-through HMD. The optical seethrough HMDs are nothing but a particular case of HUD.

The AR on handheld devices is from the video seethrough type. It is implemented on portable devices
(HHD - Hand-Held Displays) available to the general users in products such as tablets (iPad), PDAs (Blackberry) or phones (iPhones). Such devices integrate camera, display, processing capabilities and maintenance, allowing greater freedom in using these.

In the projection-based AR, images are projected on the three-dimensional surface model of the product, providing them with characteristics of its surface.

The AR with projection on the retina, (VRD Virtual Retinal Display) - developed in 1991 by Technology Laboratory Human Interfaces (HITLab) at the University of Washington - shows the virtual objects directly on the retina of the individual scanned horizontally vertically, as occurs in older TV sets (cathode ray tube). For the generation of images, low-power laser beams are used, not presenting a risk to human health.

\section{Methodology}

Methodologically, this project is divided into two complementary parts: the literature review and the experimental research.

At first, it was reviewed the literature related to the themes: usability evaluation of products and augmented reality. In the literature research were reviewed books, journals and proceedings of scientific conferences related to these themes. The goal was to determine the usability evaluation methods most appropriate for use with consumer electronics, with physical models in real environments and in parallel with virtual models. In parallel, we started the importation process of the stereoscopic HMD (VR glasses) model 3120-2 Xsight, from firma Sensics (in the USA). Augmented reality will be achieved by attaching a webcam to the HMD.

At this moment, after the literature review, we started the planning phase of the experimental research - see Figure 1. This planning phase involves: the selection of the electronic products to be evaluated; the choice of the variables of usability to be measured; details on how the evaluations will be conducted; and statistical delimitation of the experiment. A group of volunteers - potential users of products to be evaluated - should be trained to use the AR technology. 


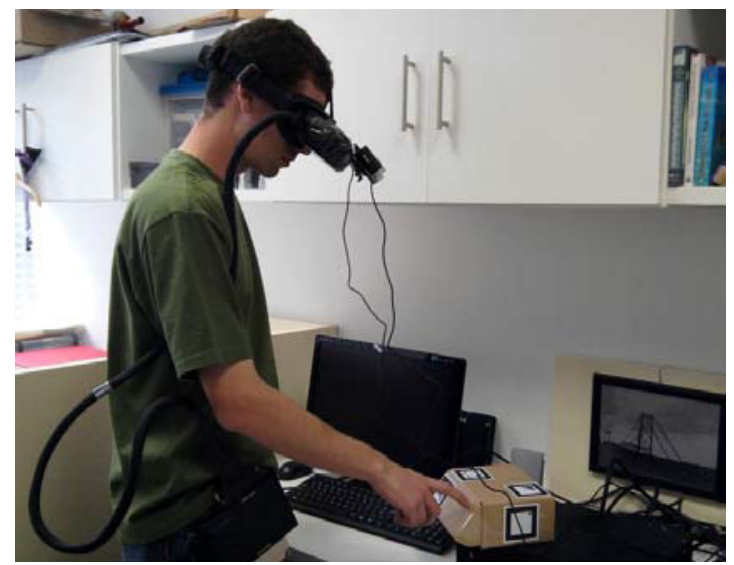

Figure 1: User interacting with an augmented prototype.

Physical prototypes are being developed for selected products and their virtual equivalents, so that they can be evaluated. Usability evaluations will be conducted at the Laboratory of Design Research of UDESC. The quantitative and qualitative data gathered in the tests will be compiled and analyzed in order to identify if there are significant differences between the results. Statistical hypothesis testing will be used for this purpose. The results of the statistical analysis will subsidize the conclusions of the research. Finally, we will analyze the possibilities for future researches on the use AR technology in product development process.

\section{Conclusion}

We have already performed an extensive literature review on the use of the AR technology along the product development process, in order to better evaluate the potential use of this technology in the design domain. We have also done a wide-ranging literature review on the evaluation methods for product usability, so as to determine the details of the experiments with users in our usability experiments. All the hardware necessary to perform the experiments were already acquired - including a high resolution (up to $1920 \times 1200$ pixels per eye) panoramic $\left(123^{\circ}\right)$ Head-Mounted Display, model xSightTM, imported from Firma Sensics in the USA - , and we are making the necessary adjustments and performing the first experiments with AR prototypes, as shown in Figure 1.

This research contributes to increase the academic knowledge about the usability (and user experience) of physical products, and also about the application of advanced interfaces in the evaluation of product interface - the application of the AR technology more specifically.

The research is being undertaken with close cooperation of an appliance company of our region. Products designed taking into account the ergonomic aspects of usability are more likely to succeed in the market. Best products give companies more competitive, which, in turn, generate jobs and income in our region.

\section{References}

[1] C. Kirner and R. Tori. Fundamentos de realidade aumentada, in: Fundamentos e tecnologia de realidade virtual e aumentada R. Tori, C. Kirner and R. Siscouto, ed., SBC, Porto Alegre, 2006, pp. 2-21.

[2] C.M. Karat, A comparison of user interface evaluation methods. John Wiley \& Sons, New York, 1994.

[3] H. Rozenfeld, F.A. Forcellini, D.C. Amaral et. al., Gestão de desenvolvimento de produtos: uma referência para a melhoria do processo, Saraiva, São Paulo, 2006.

[4] International Organization for Standardization, ISO 9241-11: Ergonomic requirements for office work with visual display terminals (VDTs) - Part 11: Guidance on usability. 1998.

[5] J. Nielsen, Usability engineering. Academic Press, San Diego, 1993.

[6] J. Ye, S. Badiyani, V. Raja and T. Schlegel, Applications of virtual reality in product design evaluation, in: Humancomputer interaction: HCI applications and service, J. A. Jacko, ed., Springer Verlag, Berlin, 2007, pp. 1190-1199

[7] M. Baxter, Projeto de produto: guia prático para o design de novos produtos, 2. ed. rev., Edgard Blucher, São Paulo, 2000.

[8] N.A. Stanton and M.S. Young, A guide to methodology in ergonomics: designing for human use, Taylor \& Francis, London, 1999.

[9] N. Back, A. Ogliari, A. Dias and J.C. Silva. Projeto integrado de produtos: planejamento, concepção e modelagem. Manole, Rio de Janeiro, 2008.

[10]O. Bimber and R. Raskar, Modern approaches to augmented reality, in: Proceedings of Siggraph 2005, Los Angeles, 2005.

[11]P.W. Jordan, An introduction to usability. Taylor \& Francis, London, 1998.

[12]R.T. Azuma, A survey of augmented reality. Presence: teleoperators and virtual environments, 6/4 (1997), 355-385. 